\title{
Desenvolvimento de um aplicativo android para a análise do circuito de Chua-Matsumoto
}

\author{
Development of an android application for the analysis of the Chua-Matsumoto circuit
}

\author{
Tiago Carvalho Martins*
}

Faculdade de Física, Instituto de Ciências Exatas, Universidade Federal do Sul e Sudeste do Pará, Marabá, PA, Brasil

Recebido em 27 de julho de 2015. Aceito em 28 de novembro de 2015

\begin{abstract}
Um aplicativo Android para a análise do circuito de Chua-Matsumoto foi desenvolvido. Este aplicativo fornece a trajetória de solução para o sistema dinâmico do circuito com condição inicial $\left(x_{0}, y_{0}, z_{0}\right)$. O sistema de equações diferenciais não-lineares é resolvido pelo método de Runge-Kutta-Fehlberg. O aplicativo possui três telas de entrada de dados: uma para as condições iniciais $\left(x_{0}, y_{0}, z_{0}\right)$, uma para os parâmetros do sistema $(\alpha, \beta, a, b)$, e uma para os parâmetros do método $\left(t_{\max }, h_{\text {max }}, h_{\text {min }}\right.$, tol $)$. Há uma tela utilizada para traçar as curvas das coordenadas $x, y$ e $z$ como funções do tempo. Também, há uma tela utilizada para traçar as projeções da trajetória nos planos $x y, x z$ e $y z$ e para exportar todos os dados para um arquivo txt. Além disso, há uma tela utilizada para exibir os expoentes de Lyapunov calculados pelo aplicativo.

Palavras-chave: circuito de Chua-Matsumoto, sistemas dinâmicos, atratores caóticos, aplicativos Android.
\end{abstract}

Abstract: An Android application for the analysis of the circuit of Chua-Matsumoto was developed. This application provides the solution trajectory to the dynamical system of the circuit with the initial condition $\left(x_{0}, y_{0}, z_{0}\right)$. The system of nonlinear differential equations is solved by Runge-Kutta-Fehlberg method. The application has three data entry screens: one for the initial conditions $\left(x_{0}, y_{0}, z_{0}\right)$, one for the parameters of the system $(\alpha, \beta, a, b)$, and one for the parameters of the method $\left(t_{\max }, h_{\max }, h_{\min }, t o l\right)$. There is a screen used to plot the curves of the coordinates $x, y$ and $z$ as functions of the time. Also, there is a screen used to plot the projections of the trajectory into the $x y, x z$ and $y z$ planes and to export all data to a txt file. Furthermore, there is a screen used to display the Lyapunov exponents calculated by the application.

Keywords: circuit of Chua-Matsumoto, dynamical systems, chaotic attractors, Android applications.

\section{Introdução}

A ideia de caos relaciona-se à existência de respostas imprevisíveis para um sistema determinístico. Todavia, não existe uma definição única para o caos. Pode-se afirmar que um sistema que apresenta o mesmo comportamento dinâmico complexo do mapa de ferradura de Smale (apresentar uma infinidade contável de órbitas periódicas de período arbitrariamente grande, uma infinidade incontável de órbitas não-periódicas, órbita densa, e sensibilidade às condições iniciais) é caótico.

Durante muitos anos não se soube se o caos representava apenas um conceito matemático, materializado na forma de erros de aproximação nos

*Endereço de correspondência: tiagocm@unifesspa.edu.br. cálculos numéricos das equações que descrevem sistemas físicos. Na década de 1980, o circuito de ChuaMatsumoto possibilitou a visualização de um atrator caótico na tela do osciloscópio, corroborando experimentalmente que o caos representa o comportamento dinâmico de sistemas físicos reais [1]. A importância de estudar o circuito de Chua-Matsumoto está relacionada à maior variedade de fenômenos que ele apresenta, os quais têm sido verificados experimentalmente em laboratório, em comparação com outros sistemas comumente estudados, como Rössler ou Lorenz [2]. A criptografia baseada em caos é uma possível aplicação prática do circuito de Chua-Matsumoto [3].

O uso de dispositivos que utilizam a plataforma Android encontra-se disseminado entre os estudantes 
de todos os níveis, trazendo ao professor o desafio de incorporar tais dispositivos ao ensino, em vez de simplesmente condená-los. O presente trabalho foi desenvolvido nesse sentido. Nele apresenta-se o desenvolvimento de um aplicativo em plataforma Android (executável em um Smartphone) para simulações computacionais do circuito de Chua-Matsumoto. O aplicativo é gratuito e está disponível no endereço eletrônico http://www.amazon.com.au/Circuit-ofChua-Matsumoto-and-Chaos.

$\mathrm{O}$ aplicativo soluciona o circuito de ChuaMatsumoto, para uma dada condição inicial $\left(x_{0}\right.$, $\left.y_{0}, z_{0}\right)$, através do método Runge-Kutta-Fehlberg. É possível escolher os valores das condições iniciais $\left(x_{0}, y_{0}\right.$ e $\left.z_{0}\right)$, dos parâmetros do circuito $(\alpha, \beta, a$ e $b)$, assim como, os parâmetros do método $\left(t_{\max }, h_{\max }\right.$, $\left.h_{\text {min }}, t o l\right)$. É possível plotar os gráficos das coordenadas $x, y$ e $z$ em função do tempo. Também há a possibilidade de visualização das projeções da trajetória nos planos $x y, x z$ e $y z$. É possível realizar o cálculo dos expoentes de Lyapunov. Além disso, pode-se exportar os dados para arquivos no formato txt, os quais são armazenados na pasta Documents.

Na seção 2, o objeto de estudo do aplicativo, o circuito de Chua-Matsumoto, é descrito. A seção 3 contém alguns conceitos da teoria de sistemas dinâmicos, os quais são necessários para que o usuário seja capaz de entender os resultados gerados pelo aplicativo. A seção 4 descreve o aplicativo e apresenta resultados obtidos com o aplicativo em um Smartphone, os quais são comparados com simulações computacionais executadas em um computador de mesa (desktop) rodando o software MATLAB. A seção 5 é a conclusão do trabalho.

\section{Modelo do circuito de Chua-Matsu- moto}

O circuito mostrado na Fig. 1 é conhecido na literatura como circuito de Chua-Matsumoto, o qual é constituído de um indutor de indutância $L$, um

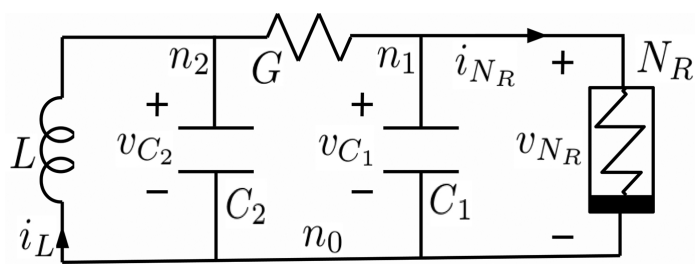

Figura 1: Circuito de Chua-Matsumoto. resistor de condutância $G$, dois capacitores de capacitâncias $C_{1}$ e $C_{2}$, e um resistor não-linear, $N_{R}$, conhecido como diodo de Chua. Os parâmetros dos componentes de circuito, $C_{1}, C_{2}, G, L$, são números reais positivos.

O diodo de Chua é um dispositivo não-linear, formado por três regiões lineares $\left(v_{N_{R}}<-B_{P}\right.$, $-B_{P} \leq v_{N_{R}} \leq B_{P}$ e $\left.v_{N_{R}}>B_{P}\right)$ de resistências negativas, cuja curva característica corrente-voltagem é dada por

$$
\begin{gathered}
i_{N_{R}}\left(v_{N_{R}}\right)=G_{b} v_{N_{R}}+ \\
\left(G_{a}-G_{b}\right)\left(\left|v_{N_{R}}+B_{P}\right|-\left|v_{N_{R}}-B_{P}\right|\right),
\end{gathered}
$$

em que $B_{P}$ é a tensão de limiar. Se o valor absoluto da tensão nos terminais de $N_{R}, v_{N_{R}}$, excede a tensão de limiar, a inclinação da curva característica é $G_{b}$; caso contrário, a inclinação da curva característica é $G_{a}$ (ver Fig. 2).

\subsection{Modelo em coordenadas adimensionais}

O circuito de Chua-Matsumoto é modelado matematicamente, em coordenadas adimensionais, através do sistema de equações diferenciais ordinárias tridimensional

$$
\begin{aligned}
\dot{x} & =\alpha(y-x-f(x))=h_{1}(x, y, z), \\
\dot{y} & =x-y+z=h_{2}(x, y, z), \quad \alpha, \beta \in \mathbb{R}^{+} \\
\dot{z} & =-\beta y=h_{3}(x, y, z) .
\end{aligned}
$$

Em que a função $f(x)$ é dada por

$$
\begin{gathered}
f(x)=b x+ \\
\frac{1}{2}(a-b)(|x+1|-|x-1|) \quad a, b \in \mathbb{R} .
\end{gathered}
$$

As correntes $i_{C_{1}}$ e $i_{C_{2}}$ fluem, respectivamente, através dos capacitores $C_{1}$ e $C_{2}$. As tensões nos

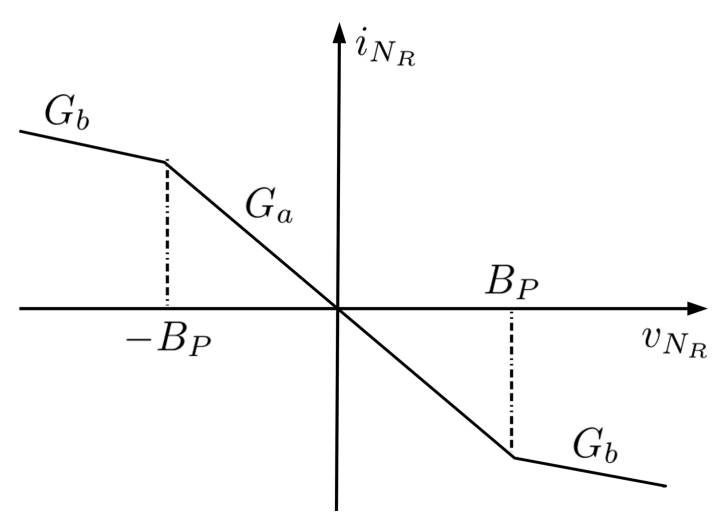

Figura 2: Curva característica corrente-voltagem do diodo de Chua. 
terminais desses capacitores são $v_{C_{1}}$ e $v_{C_{2}}$, como ilustrado na Fig. 1. Além disso, $v^{C^{1}}$ é igual a $v^{N^{R}}$, pois $C_{1}$ e $N_{R}$ possuem os mesmos terminais. A capacitância mede a capacidade de armazenamento de energia elétrica, assim como, a oposição à variação de tensão (inércia de tensão) nos terminais de um capacitor, quando atravessado por uma corrente elétrica; o que é expresso matematicamente como

$$
i_{C_{1}}=C_{1} \frac{d v_{C_{1}}}{d t}=C_{1} \frac{d v_{N_{R}}}{d t}, i_{C_{2}}=C_{2} \frac{d v_{C_{2}}}{d t} .
$$

A corrente $i_{G}$ flui através do resistor de condutância $G$. Pela primeira Lei de Ohm, tem-se

$$
i_{G}=G\left(v_{C_{2}}-v_{C_{1}}\right)=G\left(v_{C_{2}}-v_{N_{R}}\right) .
$$

Aplicando a lei de Kirchhoff das correntes (conservação de carga) respectivamente aos nós $l_{1}$ e $l_{2}$, obtém-se

$$
\begin{gathered}
i_{C_{1}}=i_{G}-i_{N_{R}} \Rightarrow \\
C_{1} \frac{d}{d t} v_{N_{R}}=G\left(v_{C_{2}}-v_{N_{R}}\right)-i_{N_{R}}, \\
i_{C_{2}}=i_{L}-i_{G} \Rightarrow \\
C_{2} \frac{d}{d t} v_{C_{2}}=i_{L}-G\left(v_{C_{2}}-v_{N_{R}}\right) .
\end{gathered}
$$

Aplicando a Lei de Kirchhoff das tensões (conservação de energia) à malha constituída pelo indutor $L$ e pelo capacitor $C_{2}$, obtém-se a equação

$$
L \frac{d}{d t} i_{L}=-v_{C_{2}} .
$$

As variáveis $v_{N_{R}}, i_{N_{R}}, v_{C_{2}}, i_{L}, t$, presentes nas Eqs. (6), (7) e (8) podem ser trocadas por variáveis adimensionais $x, y, z, \tau$, utilizando as fórmulas

$$
\begin{aligned}
& v_{N_{R}}=x B_{P}, i_{N_{R}}=\left(G B_{P}\right) f(x), \\
& v_{C_{2}}=y B_{P}, i_{L}=z B_{P} G, t=\frac{C_{2}}{G} \tau \Rightarrow \\
& \frac{d}{d \tau}=\frac{C_{2}}{G} \frac{d}{d t} .
\end{aligned}
$$

Realizando essas mudanças de coordenadas, obtém-se o sistema tridimensional mostrado na Eq. (2), onde $\alpha$ e $\beta$ são dados por

$$
\alpha=\frac{C_{2}}{C_{1}}, \beta=\frac{C_{2}}{L G^{2}} .
$$

A seu turno, a Eq. (3) pode ser obtida substituindo a Eq. (9) na Eq. (1), onde

$$
a=\frac{G a}{G}, b=\frac{G b}{G} \text {. }
$$

\subsection{Implementação do diodo de Chua}

O diodo de Chua não é vendido comercialmente, mas pode ser emulado com componentes convencionais [4 8. Na Ref. [5], Matsumoto implementou um diodo de Chua com um amplificador operacional (UA741), um par de diodos, sete resistores e uma fonte de alimentação DC de $\pm 9 \mathrm{~V}$, de acordo com o esquema de circuito mostrado na Fig. 3, resultando em um componente $N_{R}$ com a curva característica da Eq. (1), com os seguintes valores: $G a \approx-0,8 \mathrm{~mA} / \mathrm{V}$, $G b \approx-0,5 \mathrm{~mA} / \mathrm{V}$ e $\pm B p= \pm 1 \mathrm{~V}$.

O diodo de Chua também pode ser obtido através de componentes nanoeletrônicos denominados de memoristores 9 11]. Embora o conceito de memoristor tenha sido desenvolvido por Leon Chua no início da década de 1970, o primeiro memoristor foi construido por pesquisadores da HP Labs apenas em 2008, e ficou conhecido como memoristor da HP 13. Em 2012, um circuito caótico baseado em memoristores HP foi publicado 14.

\section{Sistemas dinâmicos}

As variáveis $x(t), y(t)$ e $z(t)$, cujas derivadas temporais aparecem no lado esquerdo das equações diferenciais na Eq. (2), são as variáveis de estado do sistema. Como as variáveis de estado variam com o tempo, o sistema é designado como sistema dinâmico. As variáveis de estado formam o vetor de estado dado por

$$
\mathbf{r}(t)=\left[\begin{array}{c}
x(t) \\
y(t) \\
z(t)
\end{array}\right] \equiv(x, y, z)
$$

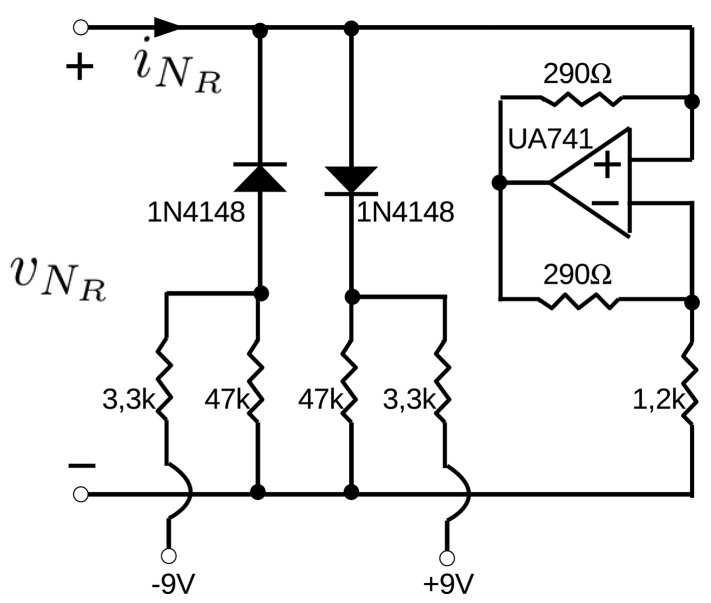

Figura 3: Esquema do circuito do diodo de Chua implementado por Matsumoto [5]. 
As variáveis de um sistema dinâmico que não representam variáveis de estado; como $\alpha, \beta, a$ e $b$; recebem a denominação de parâmetros do sistema. Se esses parâmetros não variam com o tempo $t$, o sistema é dito a parâmetros fixos.

Pode-se pensar no vetor de estado $\mathbf{r}(t)$ como o vetor posição de uma partícula, em que as variáveis $x, y$ e $z$ representam as coordenadas da posição. Uma trajetória é descrita pela partícula com o passar do tempo $t$.

O espaço de fases (ou espaço de estados) é o conjunto de todas as posições $\mathbf{r}(t) \operatorname{com} t \in \mathbb{R}$. Para a Eq. (2), o espaço de fases é o $\mathbb{R}^{3}$. A fim de solucionar o sistema, deve-se fornecer, em um certo instante de tempo $t_{0}$, o vetor de estado $\mathbf{r}_{0}=\mathbf{r}\left(t_{0}\right)$. Uma solução é uma trajetória no espaço de fases, iniciada em $\mathbf{r}_{0}$. Esse problema é conhecido como problema de valor inicial com condição inicial $\mathbf{r}_{0}$. Para garantir a existência e unicidade da solução de um problema de valor inicial, as funções $h_{i}$ e $\partial h_{i} / \partial x_{i}(i=1,2,3)$ devem ser contínuas.

Um sistema de equações diferenciais é dito autônomo, se a variável independente $t$ não aparece explicitamente nas equações. Portanto, o sistema (2) é autônomo. Um sistema não-autônomo de ordem $n, \dot{\mathbf{r}}(t)=\mathbf{h}(\mathbf{r}(t), t)$, pode ser transformado em um sistema autônomo de ordem $n+1, \dot{\mathbf{r}}^{\prime}(t)=\mathbf{h}\left(\mathbf{r}^{\prime}(t)\right)$, substituindo o vetor de estado $\mathbf{r}(t)$ pelo vetor de estado $\mathbf{r}^{\prime}(t)=(\mathbf{r}(t), t)$.

Para $x \geq 1,|x-1|=x-1$ e $|x+1|=x+1$; para $x \leq-1,|x-1|=-x+1$ e $|x+1|=-x-1$; para $|x| \leq 1,|x-1|=-x+1$ e $|x+1|=x+1 ;$ portanto, a Eq. (3) pode ser reescrita como

$$
f(x)=\left\{\begin{array}{c}
b x+a-b, \quad x \geq 1 \\
a x, \quad|x| \leq 1 \\
b x-a+b, \quad x \leq-1
\end{array}\right.
$$

evidenciando a existência de três diferentes domínios para as soluções da Eq. (2), a saber, $D_{-1}=\left\{(x, y, z) \in \mathbb{R}^{3} \mid x \leq-1\right\}, D_{1}=\{(x, y, z) \in$ $\left.\mathbb{R}^{3} \mid x \geq 1\right\}$ e $D_{0}=\left\{(x, y, z) \in \mathbb{R}^{3} \mid-1 \geq x \leq 1\right\}$. O plano $U_{-1}=\left\{(x, y, z) \in \mathbb{R}^{3} \mid x=-1\right\}$ é a fronteira entre $D_{-1}$ e $D_{0}$, e o plano $U_{1}=\{(x, y, z) \in$ $\left.\mathbb{R}^{3} \mid x=1\right\}$ é a fronteira entre $D_{1}$ e $D_{0}$.

No domínio $D_{-1}, f(x)=b x-a+b$, então $\dot{x}=\alpha(y-x-f(x))=(\alpha b-\alpha) x+\alpha y+\alpha(a-b)$, portanto, o sistema de equações pode ser escrito na forma matricial como

$$
\begin{gathered}
{\left[\begin{array}{c}
x(t) \\
\dot{y(t)} \\
z \dot{(t)}
\end{array}\right]=} \\
{\left[\begin{array}{ccc}
-\alpha \nu-\alpha & \alpha & 0 \\
1 & -1 & 1 \\
0 & -\beta & 0
\end{array}\right]\left[\begin{array}{l}
x(t) \\
y(t) \\
z(t)
\end{array}\right]+\left[\begin{array}{l}
p \\
0 \\
0
\end{array}\right]}
\end{gathered}
$$

onde $p=\alpha(a-b)$ e $\nu=b$. Definindo a matriz $\overleftrightarrow{A}$ e o vetor coluna $\mathbf{B}$ dados por

$$
\overleftrightarrow{A}=\left[\begin{array}{ccc}
-\alpha \nu-\alpha & \alpha & 0 \\
1 & -1 & 1 \\
0 & -\beta & 0
\end{array}\right], \mathbf{B}=\left[\begin{array}{l}
p \\
0 \\
0
\end{array}\right]
$$

é possível escrever a equação matricial 15 como

$$
\dot{\mathbf{r}}(t)=\overleftrightarrow{A} \mathbf{r}(t)+\mathbf{B}
$$

em que o vetor $\mathbf{B}$ é chamado de entrada do sistema, o vetor $\mathbf{r}$ é chamado de saída do sistema e a matriz $\overleftrightarrow{A}$ é chamada de matriz do sistema.

No domínio $D_{1}, f(x)=b x+a-b$, então $\dot{x}=\alpha(y-x-f(x))=(-\alpha b-\alpha) x+\alpha y+\alpha(-a+b)$, portanto, o sistema de equações pode ser escrito na forma matricial através da Eq. 15 $\operatorname{com} p=-a+b$ e $\nu=b$.

No domínio $D_{0}, f(x)=a x$, então $\dot{x}=\alpha(y-x-$ $f(x))=(-\alpha a-\alpha) x+\alpha y$, portanto, o sistema de equações pode ser escrito na forma matricial através da Eq. (15) com $p=0$ e $\nu=a$.

O sistema dinâmico (2) é contínuo, haja vista o vetor de estado $\mathbf{r}(t)$ variar continuamente com o tempo $t$. Um sistema é discreto quando há um conjunto de instantes de tempo $\left\{t_{0}, t_{1}, t_{2}, \ldots, t_{j}, \ldots\right\}$, com $j \in \mathbb{N}$, fora dos quais nunca ocorrem variações no vetor de estado.

O princípio da superposição estabelece que, se $\mathbf{r}_{\mathbf{1}}(t)$ é a saída produzida pela entrada $\mathbf{B}_{\mathbf{1}}(t)$ e $\mathbf{r}_{\mathbf{2}}(t)$ é a saída produzida pela entrada $\mathbf{B}_{\mathbf{2}}(t)$, então

$$
\mathbf{B}_{\mathbf{T}}=k_{1} \mathbf{B}_{\mathbf{1}}+k_{2} \mathbf{B}_{\mathbf{2}} \Rightarrow \mathbf{r}_{\mathbf{T}}=k_{1} \mathbf{r}_{\mathbf{1}}+k_{2} \mathbf{r}_{\mathbf{2}},
$$

onde $k_{1}$ e $k_{2}$ são constantes. Um sistema é dito linear quando obedece ao princípio da superposição, caso contrário, é dito não-linear. Malgrado o sistema (2) para o domínio $\mathbb{R}^{3}$ seja não-linear, ele assume a forma linear 17) para os domínios $D_{-1}, D_{0}$ e $D_{1}$, o que pode ser demonstrado utilizando a expressão (18).

Diz-se que um sistema é dissipativo, quando o volume que ele ocupa no espaço de fase contrai-se 
com o passar do tempo, o que pode ser expresso através da equação

$$
\nabla \cdot \mathbf{h}<0 \Rightarrow \frac{\partial h_{1}}{\partial x}+\frac{\partial h_{2}}{\partial y}+\frac{\partial h_{3}}{\partial z}<0 .
$$

Portanto, $-\alpha(b+1)-1<0$ e $-\alpha(a+1)-1<0$ são as condições para que o circuito de Chua-Matsumoto seja dissipativo em $D_{-1} \cup D_{1}$ e $D_{0}$, respectivamente.

\subsection{Pontos de equilíbrio e estabilidade no sentido de Lyapunov}

A análise do sistema nos seus pontos de equilíbrio (ou pontos fixos) é importante para estudar a estabilidade do sistema. Os pontos de equilíbrio são aqueles em que a partícula está parada, ou seja, se a partícula encontra-se no ponto de equilíbrio $\mathbf{r} * \mathrm{em}$ um certo instante de tempo $t^{*}$, então a partícula encontrar-se-á no mesmo ponto $\mathbf{r}^{*}$ para qualquer $t>t^{*}$. Matematicamente, pode-se dizer que pontos de equilíbrio $\mathbf{r}^{*}$ são aqueles para os quais

$$
\mathbf{h}\left(\mathbf{r}^{*}(t)\right)=\dot{\mathbf{r}}^{*}(t)=0 .
$$

Para sistemas de equações diferenciais que descrevem um circuito elétrico, um ponto de equilíbrio corresponde a uma solução DC (direct current) do circuito.

Um ponto regular ou ordinário é todo ponto do espaço de fases que não é ponto de equilíbrio.

Um ponto de equilíbrio $\mathbf{r}^{*}$ é assintoticamente estável se todas as trajetórias com condições iniciais contidas numa esfera de raio $\delta$ centrada em $\mathbf{r}^{*}$ tendem a $\mathbf{r}^{*}$ com o passar do tempo. Quando $\delta$ é finito o ponto de equilíbrio é localmente assintoticamente estável. Quando $\delta$ é infinito o ponto de equilíbrio é globalmente assintoticamente estável. Ambos os pontos são atratores. O conjunto das condições iniciais que tendem a um mesmo atrator formam a bacia de atração do atrator.

Um ponto de equilíbrio $\mathbf{r}^{*}$ é marginalmente estável se todas as trajetórias com condições iniciais contidas numa esfera de raio $\delta$ centrada em $\mathbf{r}^{*}$ não tendem a $\mathbf{r}^{*}$ com o passar do tempo, mas continuam dentro de uma esfera de raio $\epsilon$ centrada em $\mathbf{r}^{*}$.

Um ponto de equilíbrio $\mathbf{r}^{*}$ é instável se pelo menos uma trajetória com condição inicial contida numa esfera de raio $\delta$ centrada em $\mathbf{r}^{*}$ escapa de uma esfera de raio $\epsilon$ centrada em $\mathbf{r}^{*}$ em um tempo finito.

Os pontos de equilíbrio do sistema da Eq. (2), obtidos quando $\dot{x}=\dot{y}=\dot{z}=0$, são: $P_{-}=(-\theta, 0, \theta)$, em $x<-1 ; P_{0}=O=(0,0,0)$, em $-1<x<1$; e $P_{+}=(\theta, 0,-\theta)$ em $x>1$; em que $\theta=(b-a) /(b+$ 1) $>1$ e, portanto, a sentença $[(a<-1) \wedge(b>$ $-1)] \bigvee[(a<-1) \wedge(b>-1)]$ deve ser verdadeira, para que existam os pontos de equilíbrio $P_{-}$e $P_{+}$.

\subsection{Linearização}

Dado um ponto de equilíbrio $P$ cuja posição é $\mathbf{r}_{P}$, as expansões em séries de Taylor das componentes do vetor $\mathbf{h}=(\dot{x}, \dot{y}, \dot{z})$ da Eq. (2) são

$$
\begin{array}{r}
h_{i}(\mathbf{r}) \approx h_{i}\left(\mathbf{r}_{P}\right)+\left(x-x_{P}\right) \frac{\partial h_{i}\left(\mathbf{r}_{P}\right)}{\partial x}+ \\
+\left(y-y_{P}\right) \frac{\partial h_{i}\left(\mathbf{r}_{P}\right)}{\partial y}+\left(z-z_{P}\right) \frac{\partial h_{i}\left(\mathbf{r}_{P}\right)}{\partial z},
\end{array}
$$

onde $i=1,2,3$. Como $h_{1}(\mathbf{r})=\dot{x}, h_{1}\left(\mathbf{r}_{P}\right)=\dot{x}_{P}$, $h_{2}(\mathbf{r})=\dot{y}, h_{2}\left(\mathbf{r}_{P}\right)=\dot{y}_{P}, h_{3}(\mathbf{r})=\dot{z}$ e $h_{3}\left(\mathbf{r}_{P}\right)=\dot{z}_{P}$, as três equações obtidas podem ser expressas na forma matricial como

$$
\left[\begin{array}{c}
\dot{x}-\dot{x}_{P} \\
\dot{y}-\dot{y}_{P} \\
\dot{z}-\dot{z}_{P}
\end{array}\right] \approx\left[\begin{array}{lll}
\frac{\partial h_{1}}{\partial x} & \frac{\partial h_{1}}{\partial x} & \frac{\partial h_{1}}{\partial x} \\
\frac{\partial h_{2}}{\partial x} & \frac{\partial h_{2}}{\partial x} & \frac{\partial h_{2}}{\partial x} \\
\frac{\partial h_{3}}{\partial x} & \frac{\partial h_{3}}{\partial x} & \frac{\partial h_{3}}{\partial x}
\end{array}\right]_{P}\left[\begin{array}{l}
x-x_{P} \\
y-y_{P} \\
z-z_{P}
\end{array}\right]
$$

A Eq. 22 é uma versão linearizada do sistema (2), válida para pontos infinitesimalmente próximos do ponto $P$. A matriz do sistema linearizado é denominada de matriz jacobiana do sistema, a qual para o circuito de Chua-Matsumoto pode ser obtida a partir da Eq. (2), sendo expressa como

$$
\begin{gathered}
\overleftrightarrow{J}=\left[\begin{array}{ccc}
\frac{\partial h_{1}}{\partial x} & \frac{\partial h_{1}}{\partial y} & \frac{\partial h_{1}}{\partial z} \\
\frac{\partial h_{2}}{\partial x} & \frac{\partial h_{2}}{\partial y} & \frac{\partial h_{2}}{\partial z} \\
\frac{\partial h_{3}}{\partial x} & \frac{\partial h_{3}}{\partial y} & \frac{\partial h_{3}}{\partial z}
\end{array}\right]= \\
{\left[\begin{array}{ccc}
-\alpha \nu-\alpha & \alpha & 0 \\
1 & -1 & 1 \\
0 & -\beta & 0
\end{array}\right]}
\end{gathered}
$$

onde $\nu=b$ para $|x| \geq 1$, e $\nu=a$ para $|x| \leq 1$. A Eq. (23) coincide com a Eq. 15), pois o circuito de ChuaMatsumoto é um sistema não-linear constituído por três regiões lineares. A Eq. (22) pode ser reescrita como

$$
\dot{\mathbf{r}^{\prime}}=\overleftrightarrow{J} \mathbf{r}^{\prime}
$$

onde $\mathbf{r}^{\prime}=\mathbf{r}-\mathbf{r}_{\mathbf{P}}$.

Sabe-se que $r(t)=k e^{\lambda t}$ é solução da equação diferencial de primeira ordem $\dot{r}(t)=\lambda r(t)$. Por analogia, pode-se pensar em uma solução $\mathbf{r}^{\prime}(t)=\mathbf{v} e^{\lambda t}$ para a Eq. (24), resultando em $\lambda \mathbf{v} e^{\lambda t}=\overleftrightarrow{J} \mathbf{v} e^{\lambda t}$, logo

$$
\overleftrightarrow{J} \mathbf{v}=\lambda \mathbf{v} \Rightarrow(\lambda \overleftrightarrow{I}-\overleftrightarrow{J}) \mathbf{v}=0
$$


onde $\overleftrightarrow{I}$ é a matriz identidade de ordem 3. Nessa equação, $\lambda$ é um autovalor de $\overleftrightarrow{J}$, e v é o autovetor associado a $\lambda$. Como o circuito de Chua-Matsumoto é tridimensional, $\overleftrightarrow{J}$ possui três autovalores $\lambda_{1}, \lambda_{2}$ e $\lambda_{3}$, e três autovetores $\mathbf{v}_{\mathbf{1}}, \mathbf{v}_{\mathbf{2}}$ e $\mathbf{v}_{\mathbf{3}}$, respectivamente associados. Esses autovalores são obtidos como soluções da equação secular $\operatorname{det}(\lambda \overleftrightarrow{I}-\overleftrightarrow{J})=0$, a qual resulta em

$$
\begin{aligned}
\lambda^{3} & +((\nu+1) \alpha+1) \lambda^{2}+(\beta+\nu \alpha) \lambda \\
& +(\nu+1) \alpha \beta=0,
\end{aligned}
$$

onde $\nu=b$ para a região $D_{-1} \cup D_{1}$, que contém os pontos de equilíbrio $P_{-}$e $P_{+}$; e $\nu=a$ para a região $D_{0}$, que contém o ponto de equilíbrio $P_{0}=O$.

Se o conjunto solução da Eq. 26] não é formado por três autovalores reais, então esse conjunto possui um autovalor real $\lambda_{1}=\gamma$ e dois autovalores complexos conjugados $\lambda_{2}=\sigma+i \omega$ e $\lambda_{3}=\sigma-i \omega$. Nesse caso, se as soluções da Eq. (24) são linearmente independentes, a solução geral é a combinação linear delas, resultando na expressão

$$
\mathbf{r}^{\prime}=C_{1} \mathbf{v}_{1} e^{\gamma t}+C_{2} \mathbf{v}_{2} e^{(\sigma+i \omega) t}+C_{3} \mathbf{v}_{3} e^{(\sigma-i \omega) t},
$$

em que os autovetores $\mathbf{v}_{\mathbf{2}} \triangleq \boldsymbol{\eta}_{\boldsymbol{R}}+i \boldsymbol{\eta}_{\boldsymbol{I}}$ e $\mathbf{v}_{\mathbf{3}} \triangleq \boldsymbol{\eta}_{R}-i \boldsymbol{\eta}_{I}$ constituem um par complexo conjugado, com parte real $\boldsymbol{\eta}_{R}$ e parte imaginária $\boldsymbol{\eta}_{I}$. A matriz cujas colunas são os autovetores $\overleftrightarrow{P}=\left[\begin{array}{lll}\mathbf{v}_{1} & \mathbf{v}_{2} & \mathbf{v}_{3}\end{array}\right]$ é denominada de matriz modal.

Definindo o vetor $\mathbf{w}(t)$, dado por

$$
\mathbf{w}(t) \triangleq\left[\begin{array}{ccc}
e^{\gamma t} & 0 & 0 \\
0 & e^{(\sigma+i \omega) t} & 0 \\
0 & 0 & e^{(\sigma-i \omega}
\end{array}\right][C 1 C 2 C 3]^{T},
$$

é possível reescrever a Eq. (27) como

$$
\mathbf{r}^{\prime}(t)=\overleftrightarrow{P} \mathbf{w}(t)
$$

Derivando a Eq. (28), tem-se

$$
\dot{\mathbf{w}}(t)=\left[\begin{array}{ccc}
\gamma & 0 & 0 \\
0 & \sigma+i \omega & 0 \\
0 & 0 & \sigma-i \omega
\end{array}\right] \mathbf{w}(t) .
$$

Como $\mathbf{w}(0)=\left[\begin{array}{llll}C 1 & C 2 & C & 3\end{array}\right]^{T}$, fazendo $t=0$ na Eq. 29], obtém-se a expressão

$$
\mathbf{r}^{\prime}(0)=\overleftrightarrow{P} \mathbf{w}(0) \Rightarrow[C 1 C 2 C 3]^{T}=\overleftrightarrow{P}^{-1} \mathbf{r}^{\prime}(0)
$$

A partir da Eq. (31), são obtidos o coeficiente real $C_{1}$ e o par complexo conjugado $C_{2} \triangleq c_{C} e^{\phi_{C}}$ e
$C_{3} \triangleq c_{C} e^{-\phi_{C}}$. Reescrevendo a Eq. 27) em termos de $c_{C}, \phi_{C}, \boldsymbol{\eta}_{R}$ e $\boldsymbol{\eta}_{I}$, resulta em

$$
\begin{aligned}
& \mathbf{r}^{\prime}=C_{1} \mathbf{v}_{1} e^{\gamma t}+ \\
& 2 c_{C} e^{\sigma t}\left[\boldsymbol{\eta}_{R} \cos \left(\omega t+\phi_{C}\right)+\right. \\
& \left.\boldsymbol{\eta}_{I} \operatorname{sen}\left(\omega t+\phi_{C}\right)\right] .
\end{aligned}
$$

A trajetória $\mathbf{r}^{\prime}(t), t \in \mathbb{R}$, pode ser decomposta em duas componentes $\mathbf{r}_{R}^{\prime}$ e $\mathbf{r}_{C}^{\prime}$, dadas por

$$
\begin{aligned}
& \mathbf{r}_{R}^{\prime}=C_{1} \mathbf{v}_{1} e^{\gamma t}, \\
& \mathbf{r}_{C}^{\prime}=2 c_{C} e^{\sigma t}\left[\boldsymbol{\eta}_{R} \cos \left(\omega t+\phi_{C}\right)+\right. \\
& \left.\boldsymbol{\eta}_{I} \operatorname{sen}\left(\omega t+\phi_{C}\right)\right] .
\end{aligned}
$$

A trajetória descrita por $\mathbf{r}_{R}^{\prime}$ é uma linha reta (pois o versor de $\mathbf{r}_{R}^{\prime}$ não varia com o tempo). $\mathrm{O}$ conjunto dos pontos dessa trajetória forma o autoespaço correspondente ao autovalor real $\gamma$ no ponto fixo $\mathrm{P}$, representado por $E_{R}(P)$. Por outro lado, $\mathbf{r}_{C}^{\prime}$ é um vetor tangente ao plano gerado pelos vetores de base $\boldsymbol{\eta}_{R}$ e $\boldsymbol{\eta}_{R}$. O conjunto dos pontos desse plano forma o autoespaço correspondente aos autovalores complexos $\sigma \pm i \omega$ no ponto fixo $\mathrm{P}$, representado por $E_{C}(P)$. Nota-se que os vetores de base são mantidos quando realizadas as derivações da Eq. (33) ou da Eq. (34) com relação ao tempo $t$. Como consequência disso, se $\mathbf{r}^{\prime} \in E^{C}(P)$ (ou $\left.\mathbf{r}^{\prime} \in E^{R}(P)\right)$ em um instante $t$ $=0$, então $\mathbf{r}^{\prime} \in E^{C}(P)$ (ou $\mathbf{r}^{\prime} \in E^{R}(P)$ ) para qualquer $t>0$; isto é, uma trajetória iniciada em um autoespaço, evolui nele.

A partir da Eq. (33) conclui-se que, se $\gamma<0$, então $\mathbf{r}_{R}^{\prime} \rightarrow 0$ quando $t \rightarrow \infty$, ou seja, as trajetórias convergem assintoticamente para o ponto fixo $P$ em $E_{R}(P)$. Todavia, se $\gamma>0$, as trajetórias afastam-se de $P$.

Da Eq. (34), vê-se que, quando $\sigma<0$ e $\omega \neq 0$, as trajetórias em $E_{C}(P)$ descrevem uma espiral que converge assintoticamente para o ponto fixo $P$ (a Eq. (34) é uma elipse multiplicada por uma exponencial que decresce com o tempo), que nesse caso é um foco espiral atrativo (estável). Se $\sigma>0$ e $\omega \neq 0$, a trajetória é uma espiral que se afasta do ponto fixo $P$, o qual representa um foco espiral repulsivo (instável).

Os autovetores $\mathbf{v}_{1}$ que geram $E_{R}(P)$ são obtidos a partir da equação $(\gamma \overleftrightarrow{I}-\overleftrightarrow{J}) \mathbf{v}_{1}=0$. Fazendo $\mathbf{v}_{1}=\left(1, v_{1}^{y}, v_{1}^{z}\right)$ chega-se ao autovetor expresso como

$$
\mathbf{v}_{1}=\left(1, \frac{\gamma}{\alpha}+\nu+1,-\frac{\beta \nu}{\gamma}-\frac{\beta}{\gamma}-\frac{\beta}{\alpha}\right) .
$$


Portanto, a equação da reta $E_{R}(P)$ é dada por

$$
x=\frac{y}{\frac{\gamma}{\alpha}+\nu+1}=\frac{z}{-\frac{\beta \nu}{\gamma}-\frac{\beta}{\gamma}-\frac{\beta}{\alpha}} .
$$

Utilizando $[(\sigma \pm i \omega) \overleftrightarrow{I}-\overleftrightarrow{J}] \mathbf{v}_{2,3}=0$ com $\mathbf{v}_{2,3}=$ $\left(1, v_{2,3}^{y}, v_{2,3}^{z}\right)$, e calculando os autovetores $\boldsymbol{\eta}_{R}=$ $\left(\eta_{R}^{x}, \eta_{R}^{y}, \eta_{R}^{z}\right)=\left(\mathbf{v}_{2}+\mathbf{v}_{3}\right) / 2$ e $\boldsymbol{\eta}_{I}=\left(\eta_{I}^{x}, \eta_{I}^{y}, \eta_{I}^{z}\right)=$ $\left(\mathbf{v}_{2}-\mathbf{v}_{3}\right) /(2 i)$, encontra-se

$$
\begin{gathered}
\boldsymbol{\eta}_{R}=\left(1, \frac{\sigma+\alpha \nu+\alpha}{\alpha},-\frac{\beta \sigma^{2}+(\alpha \beta \nu+\alpha \beta) \sigma+\beta \omega^{2}}{\alpha \sigma^{2}+\alpha \omega^{2}}\right), \\
\boldsymbol{\eta}_{I}=\left(0, \frac{\omega}{\alpha}, \frac{(\beta \nu+\beta) \omega}{\sigma^{2}+\omega^{2}}\right) .
\end{gathered}
$$

Da Eq. (26) conclui-se que

$$
\begin{array}{r}
\sigma=-\frac{\gamma+\alpha \nu+\alpha+1}{2}, \\
\omega^{2}+\sigma^{2}=-2 \sigma \gamma+\alpha \nu+\beta .
\end{array}
$$

Substituindo a Eq. (38) na Eq. (37), pode-se obter, a partir do produto vetorial $\boldsymbol{\eta}_{R} \times \boldsymbol{\eta}_{I}$, um vetor normal ao plano $E_{C}(P)$. Após algumas manipulações algébricas, com a utilização de Eq. 26) $\operatorname{com} \lambda=\gamma$, chega-se à equação de $E_{C}(P)$ dada por

$$
\left(\gamma^{2}+\gamma+\beta\right) x+\alpha \gamma y+\alpha z=0 .
$$

\subsection{Forma de Jordan real}

Da Eq. (29), tem-se

$$
\mathbf{w}=\left(w_{1}, w_{2}, w_{3}\right)=\overleftrightarrow{P}^{-1} \mathbf{r}^{\prime}
$$

resultando na variável real $w_{1}$ e no par complexo conjugado $w_{2} \triangleq w_{R}+i w_{I}$ e $w_{3} \triangleq w_{R}-i w_{I}$. A matriz $\overleftrightarrow{P}$ é dada por

$$
\overleftrightarrow{P}=\left[\begin{array}{ccc}
2 & 0 & 1 \\
2 \eta_{R}^{y} & -2 \eta_{I}^{y} & v_{1}^{y} \\
2 \eta_{R}^{z} & -2 \eta_{I}^{z} & v_{1}{ }^{z}
\end{array}\right]
$$

Da Eq. (30), tem-se que

$$
\begin{array}{r}
\dot{w}_{2,3}=(\sigma \pm i \omega) w_{2,3} \\
\therefore \dot{w}_{R} \pm i \dot{w}_{I}=(\sigma \pm i \omega)\left(w_{R} \pm i w_{I}\right) \\
\therefore \dot{w}_{R}=\sigma w_{R}-\omega w_{I}, \dot{w}_{I}=\omega w_{R}+\sigma w_{I} \\
\therefore\left[\begin{array}{c}
\dot{w}_{R} \\
\dot{w}_{I} \\
\dot{w}_{1}
\end{array}\right]=\left[\begin{array}{ccc}
\sigma & -\omega & 0 \\
\omega & \sigma & 0 \\
0 & 0 & \gamma
\end{array}\right]\left[\begin{array}{c}
w_{R} \\
w_{I} \\
w_{1}
\end{array}\right]
\end{array}
$$

A matriz do sistema (42) é a forma de Jordan real do circuito de Chua-Matsumoto da Eq. (2), cuja validade restringe-se a um dos domínios lineares (as variáveis $w_{R}(t), w_{I}(t), w_{1}(t)$ obtidas em $D_{0}$ não são as mesmas obtidas em $D_{1}$ ).

Utilizando a Eq. (40), o vetor de base $\mathbf{v}_{1}$ do subespaço $E_{R}(O)$ transforma-se em ${\underset{\sim}{\mathbf{v}}}_{1}=$ $\left(w_{R}, w_{I}, w_{1}\right)=(0,0,1)$, e os vetores de base $\boldsymbol{\eta}_{R}$ e $\boldsymbol{\eta}_{I}$ do subespaço $E_{C}(O)$ transformam-se em $\underset{\sim_{R}}{\boldsymbol{\eta}}=$ $\left(w_{R}, w_{I}, w_{1}\right)=(1 / 2,0,0)$ e $\underset{\sim_{I}}{\boldsymbol{\eta}}=\left(w_{R}, w_{I}, w_{1}\right)=$ $(0,-1 / 2,0)$. Portanto, o sistema (42) é obtido a partir de (2), através de uma transformação de coordenadas que coloca $E_{R}(O)$ sobre um dos eixos, no caso $\mathrm{w}_{1}$, e $E_{C}(O)$ sobre o plano perpendicular a esse eixo, no caso $w_{1}=0$.

\subsection{Ponto fixo do tipo sela-foco e órbita ho- moclínica}

A trajetória que passa por um ponto de equilíbrio $P$ é referida como órbita homoclínica se tende a $P$ quando $t \rightarrow \infty$ e $t \rightarrow-\infty$. O ponto $P$ é denominado de ponto homoclínico. Para facilitar a compreensão, utilizar-se-á o exemplo ilustrado na Fig. 4.

Se $\sigma \gamma<0$ para um ponto de equilíbrio $O$ com autovalores $\lambda_{1}=\gamma, \lambda_{2}=\sigma+i \omega, \lambda_{3}=\sigma-i \omega$; então esse ponto é do tipo sela, pois dados os subespaços $E_{R}(O)$ e $E_{C}(O)$, se $O$ é assintoticamente estável em um deles, será instável no outro. Além disso, se $\omega \neq 0$, então as trajetórias em $E_{C}(O)$ são focos espirais. Um ponto fixo do tipo sela-foco é um ponto fixo do tipo sela com $\omega \neq 0$.

Para o ponto fixo $O$, a posição dos pontos da trajetória é $\mathbf{r}(t)=\mathbf{r}^{\prime}(t)+O=\mathbf{r}^{\prime}(t)$. Caso o ponto fixo fosse $P_{+/-}$, a posição dos pontos da trajetória seria $\mathbf{r}(t)=\mathbf{r}^{\prime}(t)+P_{+/-}$.

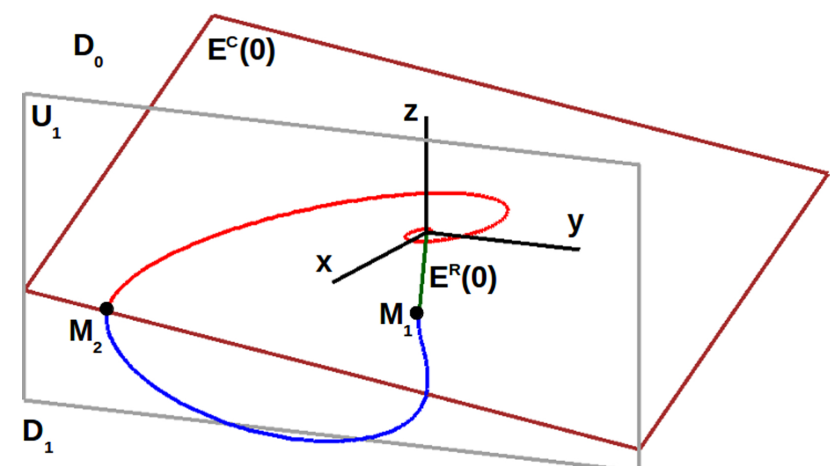

Figura 4: Órbita homoclínica no ponto fixo $O=(0,0,0)$, para os parâmetros $\alpha=11.0917459, \beta=14.2857143$, $a=-1.1428571$ e $b=-0.7142857$. A trajetória foi obtida através da Eq. (27). 
Uma órbita homoclínica é obtida no ponto fixo $\mathrm{O}=(0,0,0)$, para os parâmetros $\alpha=11.0917459$, $\beta=14.2857143, a=-1.1428571$ e $b=-0.7142857$. Os autovalores de $O$ são $\lambda_{1}=\gamma, \lambda_{2}=\sigma+i \omega$, $\lambda_{3}=\sigma-i \omega \operatorname{com} \gamma=2.835, \sigma=-1.125$ e $\omega=2.593$. Como $\sigma \gamma<0$ e $\omega \neq 0, O$ é um ponto fixo do tipo sela-foco. A trajetória mostrada na Fig. 4 foi obtida através da Eq. (27).

Na Fig. 4, a trajetória no subespaço instável $E_{R}(O)$ parte de $O$ para $M_{1}$. O ponto $M_{1}$ é a interseç̧ão $E^{R}(O) \cap U_{1}$ (obtido fazendo $x=1$ na Eq. (36)). Na região $D_{1}$ a trajetória é uma curva que parte de $M_{1}$ para $M_{2}$. O ponto $M_{2}$ pertence à reta formada pela intersecção dos planos $E^{C}(O) \cap U_{1}$. Finalmente, partindo de $M_{2}$, a trajetória descreve uma espiral que converge assintoticamente para $O$. Em síntese, a trajetória tende ao ponto fixo $O$ quando $t \rightarrow \infty$ e $t \rightarrow-\infty$, ou seja, a trajetória é uma órbita homoclínica.

Em 1986, Chua, Komuro e Matsumoto apresentaram uma prova rigorosa de que é possível encontrar órbitas homoclínicas para o circuito de Chua-Matsumoto através da escolha adequada dos parâmetros $\alpha, \beta, a$ e $b$ 15.

\subsection{Seção de Poincaré e mapa de primeiro retorno}

Dado um sistema tridimensional, pode-se analisar os pontos de intersecção entre as trajetórias e uma seção transversal às mesmas (denominada de seção de Poincaré), em vez das próprias trajetórias. Vários pontos de interseção entre uma trajetória e a seção de Poincaré são obtidos ao longo do tempo, dando origem a uma versão bidimensional discreta do sistema contínuo tridimensional. Um mapa de primeiro retorno (ou mapa de Poincaré) transforma os pontos de uma seção de Poincaré nos pontos obtidos quando a trajetória atravessa novamente a seção no mesmo sentido. Uma análise bastante completa do circuito de Chua-Matsumoto utilizando mapas de primeiro retorno é apresentada na Ref. [15].

Em Fig. 5. é mostrada uma seção de Poincaré, em $x=1$, para $\alpha=11.0917459, \beta=14.2857143, a=$ 1.1428571 e $b=-0.7142857$. No tempo $t_{0}$, há 400 pontos distanciados de 0.0001 , em torno do ponto $M_{1}$. Em um tempo $t_{1}$, posterior, os pontos evoluem para pontos próximos a $M_{2}$. Nota-se que nesse caso, em que as trajetórias não passam pelo ponto homoclínico, a trajetória não apresenta sensibilidade às condições iniciais.

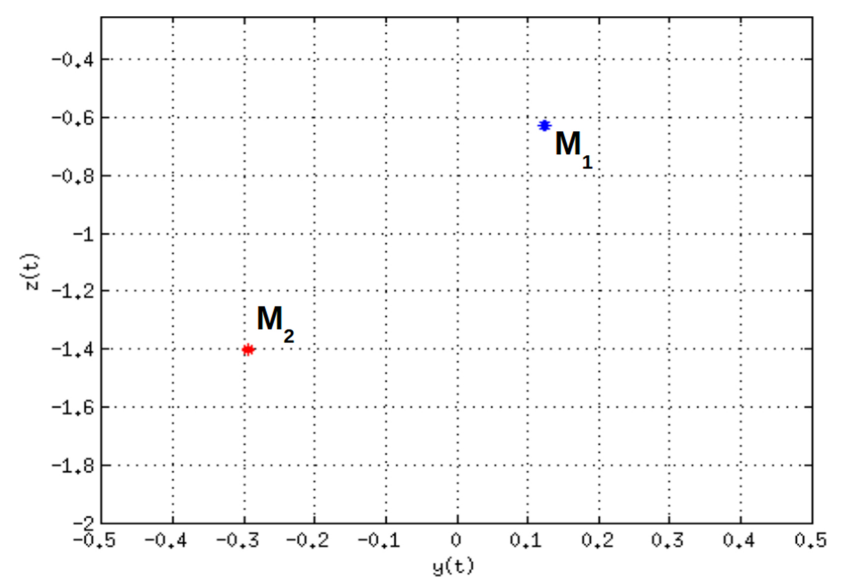

Figura 5: seção de Poincaré, em $x=1$, para $\alpha=11.0917459, \beta=14.2857143, a=-1.1428571 \mathrm{e}$ $b=-0.7142857$. No tempo $t_{0}$, há 400 pontos distanciados de 0.0001 , em torno do ponto $M_{1}$. Em um tempo $t_{1}$, posterior, os pontos evoluem para pontos próximos a $M_{2}$.

Na Fig. 6, é mostrada uma seção de Poincaré, em $x=1$, para $\alpha=11.0917459, \beta=14.2857143$, $a=-1.1428571$ e $b=-0.7142857$. A seção mostra dois instantes consecutivos de um mapa de primeiro retorno. Em $t_{0}$, há 400 pontos $(20 \times 20)$ distanciados entre si de 0.0001, em torno do ponto $M_{1}$. Em $t_{1}$, posterior, o sistema evoluiu para pontos distantes entre si. Nesse caso, em que a trajetória passa nas proximidades do ponto homoclínico $O$, a trajetória apresenta sensibilidade às condições iniciais.

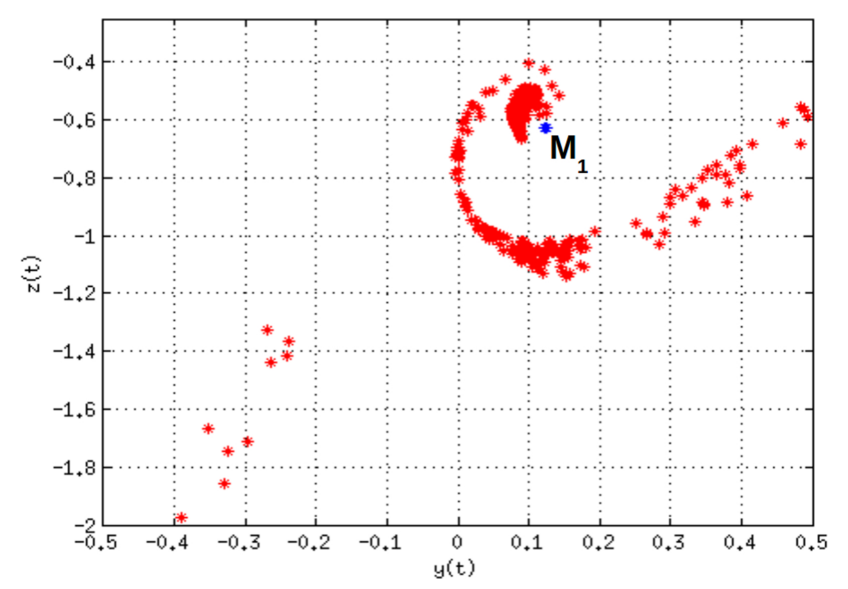

Figura 6: Seção de Poincaré, em $x=1$, para $\alpha=11.0917459, \beta=14.2857143, a=-1.1428571 \mathrm{e}$ $b=-0.7142857$. A seção mostra dois instantes consecutivos de um mapa de primeiro retorno. Em $t_{0}$, há 400 pontos $(20 \times 20)$ distanciados entre si de 0.0001 , em torno do ponto $M_{1}$. Em $t_{1}$, posterior, o sistema evoluiu para pontos distantes entre si. 


\subsection{Ferradura de Smale}

O quadrado unitário $D$ na Fig. 7 é transformado por $f$ em um objeto em forma de ferradura (ferradura de Smale). A transformação $f$ realiza a expansão, contração e dobra em três direções distintas, produzidas pela equação de evolução temporal dada por

$$
\begin{gathered}
{\left[\begin{array}{l}
x_{n} \\
y_{n}
\end{array}\right]=\left[\begin{array}{cc}
1 / 3 & 0 \\
0 & 3
\end{array}\right]\left[\begin{array}{l}
x_{n-1} \\
y_{n-1}
\end{array}\right], \text { em } H_{0},} \\
{\left[\begin{array}{l}
x_{n} \\
y_{n}
\end{array}\right]=} \\
{\left[\begin{array}{cc}
-1 / 3 & 0 \\
0 & -3
\end{array}\right]\left[\begin{array}{l}
x_{n-1} \\
y_{n-1}
\end{array}\right]+\left[\begin{array}{l}
1 \\
3
\end{array}\right], \text { em } H_{1} .}
\end{gathered}
$$

A transformação inversa $f_{-1}$ é mostrada na Fig. 8 . A medida que ocorrem sucessivas iterações, a área de intersecção da ferradura com $D$ reduz-se e dividese em um maior número de regiões desconexas, como ilustrado na Fig. 9] Dá-se o mesmo quando sucessivas

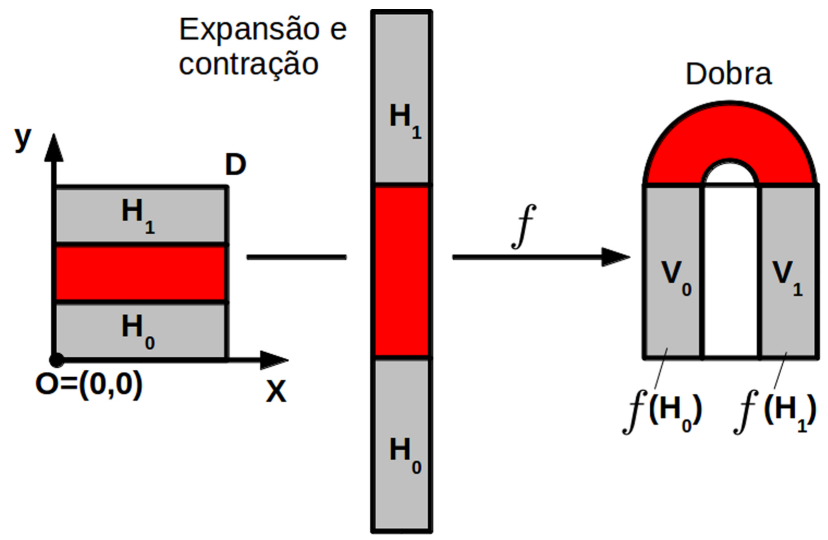

Figura 7: Expansão, contração e dobra do quadrado unitário realizadas pela transformação $f$.
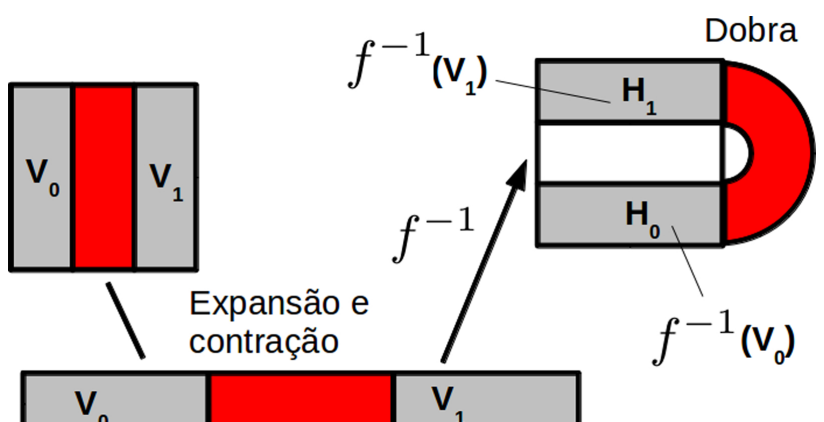

Figura 8: Expansão, contração e dobra do quadrado unitário realizadas pela transformação inversa $f^{-1}$.

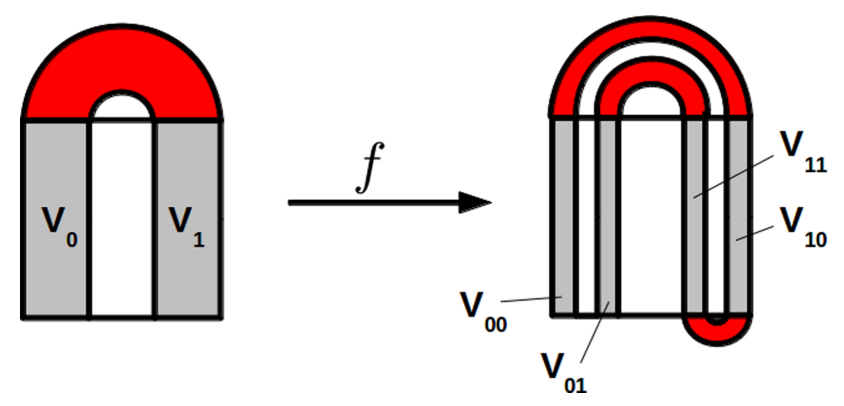

Figura 9: Aplicação de $f$ sobre $f(D)$, ou seja, $f^{2}(D)$.

iterações são retrocedidas no tempo, como ilustrado na Fig. 10.

Se todas as transformações $f$ forem realizadas sobre $D$, obter-se-á infinitos segmentos de reta unitários verticais. Para transformações inversas $f_{-1}$, obter-se-á infinitos segmentos de reta unitários horizontais. O conjunto das órbitas que permanecem em D após todas as transformações é obtido pelos pontos de intersecção entre os segmentos unitários horizontais e verticais, e é dado por

$$
\begin{aligned}
& \Lambda=\left\{\ldots f^{-1}(D) \cap D \cap f^{1}(D) \ldots\right\}= \\
& \cap_{n=-\infty}^{n=+\infty} f^{n}(D) .
\end{aligned}
$$

Em vez de analisar a evolução das órbitas, é possível analisar a evolução dos itinerários que informam em qual região de $D\left(H_{0}\right.$ ou $\left.H_{1}\right)$ a órbita encontra-se a cada iteração. Esses itinerários podem ser representados por sequências bi-infinitas $s=\left\{\ldots s_{-2} s_{-1} . s_{0} s_{1} s_{2} \ldots\right\}$, com $s_{k}=0$, se a órbita passa por $H_{0}$, e $s_{k}=1$, se órbita passa por $H_{1}$.

Pode-se definir a transformação de desvio $\sigma(s)=\left\{\ldots s_{0} . s_{1} s_{2} s_{3} \ldots\right\}$ em $\Sigma$ (conjunto de todas as sequências bi-infinitas). A transformação $\sigma$ faz cada termo $s_{\mathrm{n}}$ da sequência avançar para $s_{n+1}$, representando a lei de evolução temporal em $\Sigma$, que corresponde a $f$ em $\Lambda$. É possível provar que as conclusões obtidas para a dinâmica de $\sigma$ em $\Sigma$ também são válidas para $f$ em $\Lambda$.

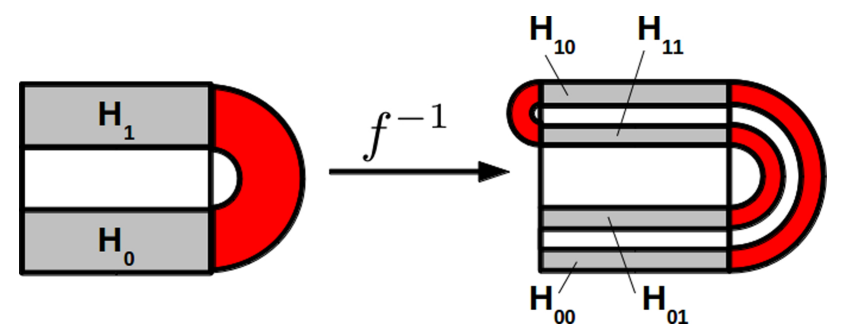

Figura 10: Aplicação de $f^{-1}$ sobre $f^{-1}(D)$, ou seja, $f^{-2}(D)$. 
Uma sequência bi-infinita $s=\left\{\ldots s_{-1} \cdot s_{0} s_{2} \ldots\right\}$ possui uma órbita periódica, se existe $T \in \mathbb{N}$ tal que $\sigma^{T}(s)=s$, onde $T$ é o período. Por exemplo, $s=\{\ldots 101101.101101 \ldots\}=\{\overline{101.101}\}$ é uma órbita periódica de período 3. Para qualquer número natural escrito na forma binária $\chi$, é possível obter uma órbita periódica $s=\{\overline{\chi \cdot \chi}\}$. Portanto, há uma infinidade contável de órbitas periódicas de período arbitrariamente grande.

A cada sequência bi-infinita $s=\left\{\ldots s_{-1} . s_{0} s_{2} \ldots\right\}$ corresponde uma sequência infinita $\bar{s}=\left\{0 . s_{0} s_{-1} s_{2} s_{-2} \ldots\right\}$. Se $s$ é não-periódica, $0 . s_{0} s_{-1} s_{2} s_{-2} \ldots$ é um número irracional; portanto, há uma infinidade incontável de órbitas não-periódicas.

A distância entre duas sequências bi-infinitas $s=\left\{\ldots s_{-2} s_{-1} . s_{0} s_{1} s_{2} \ldots\right\}$ e $s^{\prime}=\left\{\ldots s_{-2}^{\prime} s_{-1}^{\prime} . s_{0}^{\prime} s_{1} s_{2}^{\prime} \ldots\right\}$ é dada por $d\left(s, s^{\prime}\right)=\sum_{k=-\infty}^{k=+\infty} \delta_{k} / 2^{|k|}$, onde $\delta_{k}=0$, se $s_{k}=s_{k}^{\prime}$, ou $\delta_{k}=1$, se $s_{k} \neq s_{k}^{\prime}$. Afirmar que $s$ e $s^{\prime}$ estão próximos (dado um $\varepsilon>0, d\left(s, s^{\prime}\right)<\varepsilon$ ), equivale a dizer: dado um $N \in \mathbb{N}, s_{k}=s_{k}^{\prime}$ para $|k|<N$, onde $N=N(\varepsilon)$.

Uma sequência s possui órbita densa quando, dados $s^{\prime} \in \Sigma$ e $\varepsilon>0$, existe um número de iterações $T$ tal que $d\left(\sigma^{T}(s), s^{\prime}\right)<\varepsilon$. A sequência $\tilde{s}=\{\ldots\{11\}\{01\}\{1\} .\{0\}\{00\}\{10\} \ldots\}$ (formada por todas as sequências finitas com 0's e 1's) possui órbita densa, pois, após um certo número $T$ de iterações, tem-se $s_{k}=s_{k}^{\prime}$ para $|k|<N$, onde $s=\sigma^{T}(\tilde{s})$ e $N=N(\varepsilon) \in \mathbb{N}$.

A transformação $\sigma$ em $\Sigma$ possui sensibilidade às condições iniciais. Dados $s, \bar{s} \in \Sigma$ e $\varepsilon>0$, tais que $d(s, \bar{s})<\varepsilon$, ou seja, $s_{k}=\bar{s}_{k}$ para $|k|<N$; após $N$ iterações, não será mais possível afirmar que $d\left(\sigma^{N}(s), \sigma^{N}(\bar{s})\right)<\varepsilon$, pois $|k|>N$, e não será possível garantir que $s_{k}=\bar{s}_{k}$.

Como a dinâmica de $f$ em $\Lambda$ pode ser descrita em termos da dinâmica de $\sigma$ em $\Sigma$, a transformação da ferradura de Smale possui:

- Uma infinidade contável de órbitas periódicas de período arbitrariamente grande;

- Uma infinidade incontável de órbitas nãoperiódicas;

- Órbita densa;

- Sensibilidade às condições iniciais.

\subsection{Teorema de Shilnikov}

O teorema de Shilnikov foi publicado em 1965 [16]. Segundo esse teorema [15], dado um sistema tridimensional $\dot{\mathbf{r}}=h(\mathbf{r})$ contínuo e linear por partes que possui um ponto fixo do tipo sela-foco na origem com um autovalor real $\gamma$ e um par complexo conjugado de autovalores $\sigma \pm i \omega$, com $\sigma \gamma<0$ e $\omega \neq 0$; se $|\sigma|<|\gamma|$ (condição de Shilnikov) e existe uma órbita homoclínica através da origem, então existe uma ferradura de Smale próxima a essa órbita homoclínica.

É possível encontrar um sistema de coordenadas em que uma órbita homoclínica, como aquela mostrada na Fig. 4 tenha o subespaço instável $E_{R}(0)$ sobre o eixo $z$ e o subespaço estável $E_{C}(0)$ sobre o plano $x y$, como ilustrado na Fig. 11. Isso pode ser feito utilizando o conjunto dos autovetores como base, como em 42.

Um conjunto de pontos de uma seção de Poincaré tende a contrair-se para um único ponto, quando a seção é tomada cada vez mais próxima a um ponto fixo assintoticamente estável. Se em vez disso, o ponto fixo for instável, os pontos da seção afastarse-ão. Se o ponto fixo for homoclínico, a seção será contraída na direção do subespaço estável e será expandida na direção do subespaço instável. Além disso, se o o sistema for dissipativo, a seção terá de ser dobrada para que caiba no espaço de fases. Portanto, o retângulo (que é um conjunto de condições iniciais de várias trajetórias) na Fig. 4 transforma-se com as mesmas propriedades da ferradura de Smale. Nesse caso, o circuito de Chua-Matsumoto possuirá: uma infinidade contável de órbitas periódicas de período arbitrariamente grande, uma infinidade incontável de órbitas não-periódicas, órbita densa, e sensibilidade às condições iniciais.

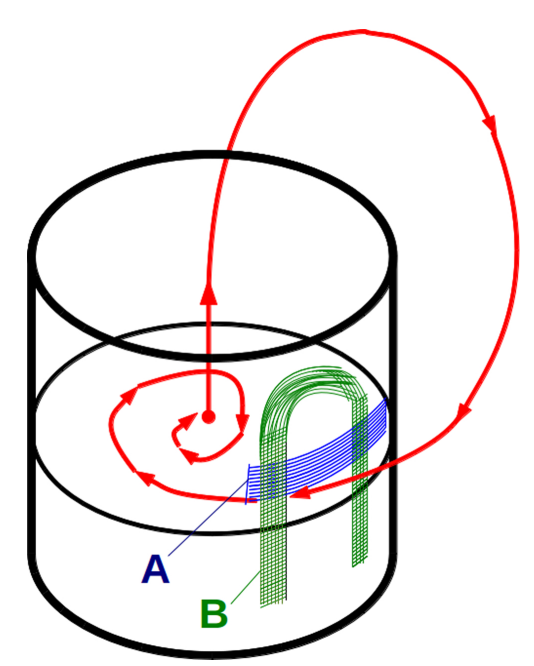

Figura 11: Ferradura de Smale próxima a uma trajetória homoclínica. 


\subsection{Expoentes de Lyapunov}

Pode-se pensar em um cubo centrado em um ponto de uma órbita homoclínica, que seja formado por pontos que representam condições iniciais. A medida que o tempo passa, os pontos desse cubo evoluem de uma forma que, a dimensão do cubo na direção da órbita deve ser mantida constante, pois caso diminuísse, convergiria para um ponto, e caso aumentasse, os pontos afastar-se-iam uns dos outros e da órbita, com o passar do tempo.

Considerando que as dimensões do cubo são $d_{j}(t)=d_{j}\left(t_{0}\right) e^{\Lambda_{j}(t-t 0)}$, onde $j=1,2,3$ e $\Lambda_{1}, \Lambda_{2}$ e $\Lambda_{3}$ são os expoentes de Lyapunov. Por exemplo, se $d_{2}(t)$ é a dimensão na direção de uma órbita homoclínica, então $\Lambda_{2}=0$, e $d_{2}(t)=d_{2}\left(t_{0}\right)$ não varia com o tempo (o expoente na direção da trajetória deve ser nulo, pois as trajetórias nunca se afastam do atrator caótico, nem tendem a um ponto fixo). Se $d_{1}(t)$ é a dimensão na direção do subespaço instável, então $\Lambda_{1}>0$, e $d_{1}(t)=d_{1}\left(t_{0}\right) e^{\Lambda_{1}(t-t 0)}$ cresce exponencialmente com o tempo. Se $d_{3}(t)$ é a dimensão na direção do subespaço estável, então $\Lambda_{3}<0$, e $d_{3}(t)=d_{3}\left(t_{0}\right) e^{\Lambda_{3}(t-t 0)}$ diminui exponencialmente com o tempo. $\mathrm{O}$ volume do cubo é

$$
\begin{array}{r}
V(t)=d_{1}(t) d_{2}(t) d_{3}(t)= \\
V\left(t_{0}\right) e^{(t-t 0) \sum_{j=1}^{3} \Lambda_{j}} .
\end{array}
$$

Um sistema caótico é dissipativo, pois as trajetórias não se afastam do atrator caótico, portanto,

$$
\sum_{j=1}^{3} \Lambda_{j}=\frac{1}{t-t 0} \ln \left(\frac{V(t)}{V\left(t_{0}\right)}\right)<0 .
$$

\section{Aplicativo Circuit of Chua and Chaos}

Nessa seção serão descritos os métodos numéricos implementados no aplicativo. Além disso, serão apresentados alguns resultados obtidos através de simulações com o programa Circuit of Chua and Chaos, realizadas em um Smartphone. Esses resultados serão comparados aos obtidos com simulações utilizando o programa MATDS (para MATLAB) ${ }^{1}$ realizadas em um computador de mesa. As liguagens utilizadas no desenvolvimento do aplicativo foram $X M L$ e Java. Todas as telas do aplicativo

\footnotetext{
${ }^{1}$ ver http://www.math.rsu.ru/mexmat/kvm/matds/
}

foram desenvolvidas em $X M L$, e toda a lógica de programação foi implementada em Java, inclusive os algoritmos do método Runge-Kutta-Fehlberg e da técnica empregada no cálculo dos expoentes de Lyapunov.

É possível fazer simulações sem realizar a entrada de dados, pois os valores $\alpha=15.0, \beta=25.58$, $a$ $=-1.14, b=-0.71 x_{0}=1.6, y_{0}=0.0, z_{0}=-1.5$ e $t_{\max }=45.0, h_{\max }=0.01, h_{\min }=1.0 \mathrm{E}-16 \mathrm{e}$ tol $=1.0 E-15$ estão previamente selecionados. A escolha de outros valores podem ser realizadas nas telas de entradas de dados. Na Fig. 12 é mostrada a tela de entrada de dados para os parâmetros do circuito de Chua. Há outras telas para escolher a condição inicial e os parâmetros do método RungeKutta-Fehlberg.

\subsection{Simulação computacional}

O método de Runge-Kutta-Fehlberg foi realizado a partir da generalização para o caso tridimensional de um algoritmo (para o caso unidimensional) apresentado na Ref. [17] (algoritmo 5.3 da p. 275). O cálculo dos expoentes de Lyapunov foi realizado com base no algoritmo apresentado na Ref. [18]. Ambos os algoritmos tiveram de ser adaptados ao caso do circuito de Chua-Matsumoto.

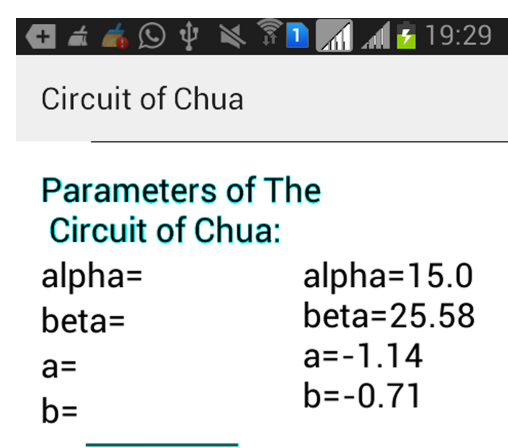

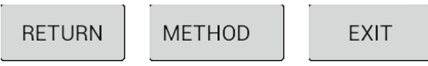

Figura 12: Tela para entrada de dados em que os parâmetros do circuito de Chua-Matsumoto $(\alpha, \beta$, a e b) podem ser escolhidos. 
O exemplo de simulação apresentado aqui utiliza os valores previamente selecionados no aplicativo, isto é

$$
\begin{aligned}
\alpha & =15.0, \beta=25.58, a=-1.14, b=-0.71, \\
x_{0} & =1.6, y_{0}=0.0, z_{0}=-1.5, \\
t_{\text {max }} & =45, h_{\max }=0.01, \\
h_{\min } & =1 E-16, \text { tol }=1 E-15 .
\end{aligned}
$$

Os parâmetros do método Runge-Kutta-Fehlberg $t_{\max }, h_{\max }, h_{\min }$ e tol representam, respectivamente, o tempo máximo de simulação, o passo temporal máximo, o passo temporal mínimo e a tolerância do método.

A tela utilizada para plotar os gráfico da projeção da trajetória no plano $x y$ está mostrada na Fig. 13. para os parâmetros mostrados na Eq. (47). Pode-se escolher também o plano $y z$ ou $x z$. A tela utilizada para plotar os gráficos das curvas $x(t)$ está mostrada na Fig. 14 . Pode-se plotar também $y(t)$ e $z(t)$ em função do tempo.

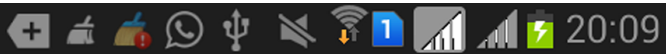

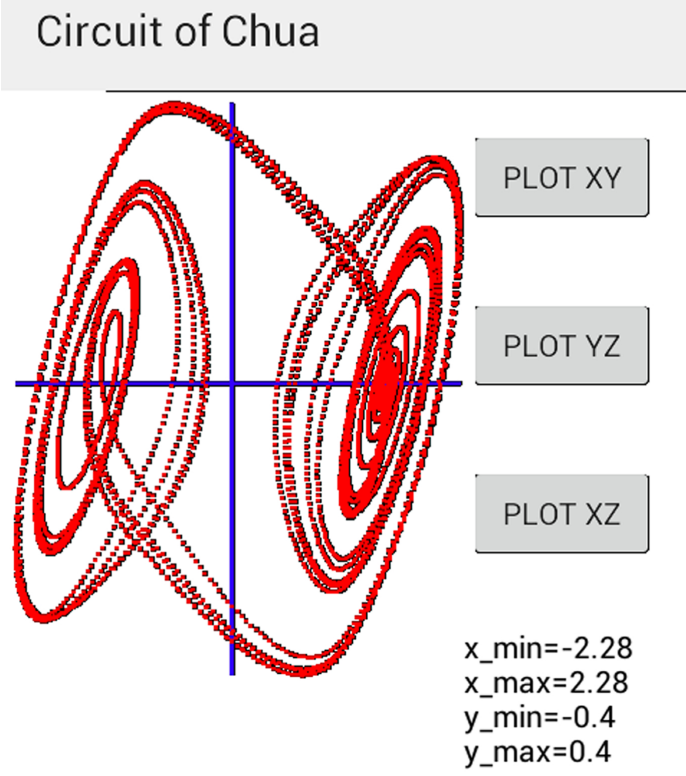

Export txt

No export

\section{RETURN}

Figura 13: Tela de plotagem das projeções da trajetória. Foi plotada a projeção da trajetória no plano $x y$, para os parâmetros mostrados na Eq. (47).

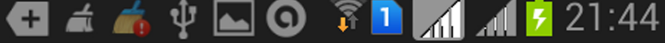

\section{Circuit of Chua}

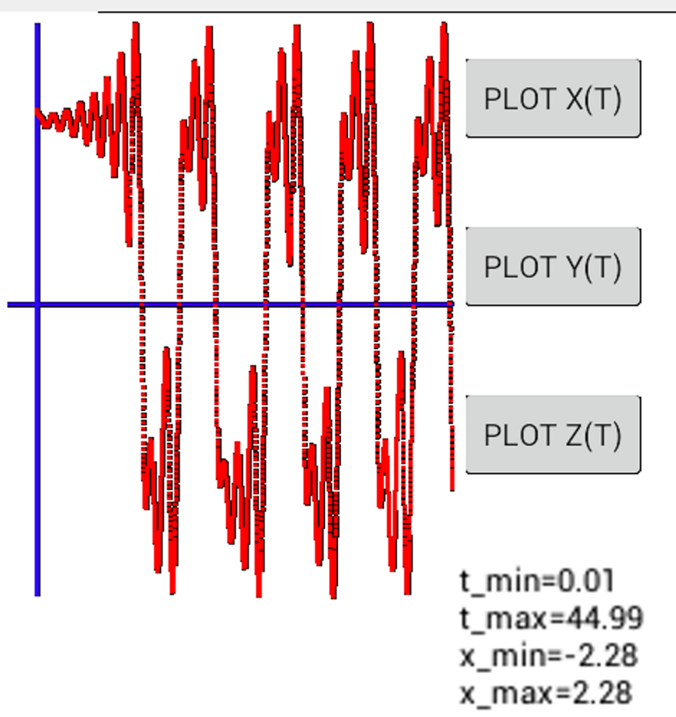

Export txt

No export

RETURN

Figura 14: Tela de plotagem das curvas em função de $t$. Foi plotado o gráfico da curva $x(t)$, para os parâmetros mostrados na Eq. (47).

Na Fig. 15 é mostrada a tela de cálculo dos expoentes de Lyapunov, a qual apresenta os valores $\Lambda_{1}=0.36, \Lambda_{2}=0.00$, e $\Lambda_{3}=-4.15$, para os parâmetros mostrados na Eq. (47). A soma dos expoentes é negativa, indicando que o sistema é dissipativo. Como visto anteriormente, esses valores correspondem a uma solução caótica. Utilizando o aplicativo $M A T D S$, foram obtidos os valores $\Lambda_{1}=0.39$, $\Lambda_{2}=0.00$, e $\Lambda_{3}=-4.12$. Vê-se que esses resultados estão de acordo.

Os pontos de equilíbrio, para os parâmetros da Eq. (47), são $P_{+}=(1.48,0,-1.48), P_{0}=(0,0,0)$ e $P_{-}=(-1.48,0,1.48)$. Os autovalores de $P_{0}$ são $\lambda_{1}=-1.1259+3.8418 i, \lambda_{2}=-1.1259-3.8418 i$ e $\lambda_{3}=3.3517$. Os autovalores de $P_{+}$e $P_{-}$são $\lambda_{1}=0.31038+4.30581 i, \lambda_{2}=0.31038-4.30581 i$ e $\lambda_{3}=-5.9708$. Nota-se que todos os pontos de equilíbrio são do tipo sela-foco e obedecem à condição de Shilnikov. 


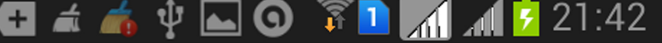

Circuit of Chua

\section{RETURN}

EXIT

0.36230085102763937

$-5.828676615160244 \mathrm{E}-4$

$-4.147520562377864$

\section{LYAPUNOV EXPONENT}

CALCULATION

Figura 15: Tela de cálculo dos expoentes de Lyapunov, para os parâmetros mostrados na Eq. 47.

Na Fig. 16 é mostrada a trajetória do atrator de rolo duplo (double-scroll) para o circuito de Chua-Matsumoto com os parâmetros mostrados na Eq. (47). Os resultados obtidos com o aplicativo Circuit of Chua and Chaos foram comparados aos obtidos com o programa MATDS. Os resultados são coincidentes.

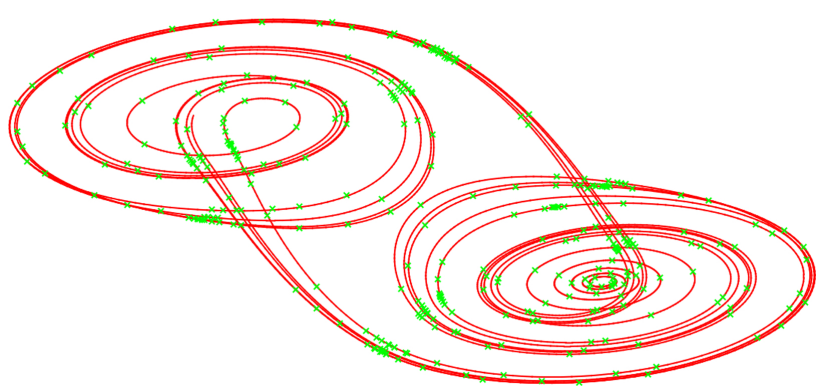

Figura 16: Atrator de rolo duplo (double-scroll) para o circuito de Chua-Matsumoto com os parâmetros mostrados na Eq. (47). Os resultados obtidos com o aplicativo Circuit of Chua and Chaos são comparados aos obtidos com o programa MATDS. Os resultados são coincidentes.
Nas Figs. 17, 18 e 19 são mostrados, respectivamente, os gráficos das curvas de $x(t), y(t)$ e $z(t)$ em função de $t$. Todos os resultados estão em concordância.

\section{Conclusão}

Realizar simulações com o circuito de ChuaMatsumoto é uma forma eficiente de tornar mais atrativo o estudo de sistemas dinâmicos (uma disciplina que em geral é ofertada tanto para os cursos de engenharia quanto para os cursos de física e matemática), auxiliando no aprendizado de conceitos relacionados à disciplina. $\mathrm{O}$ uso da plataforma Android possui algumas vantagens em termos da realização de simulações com relação às outras tec-

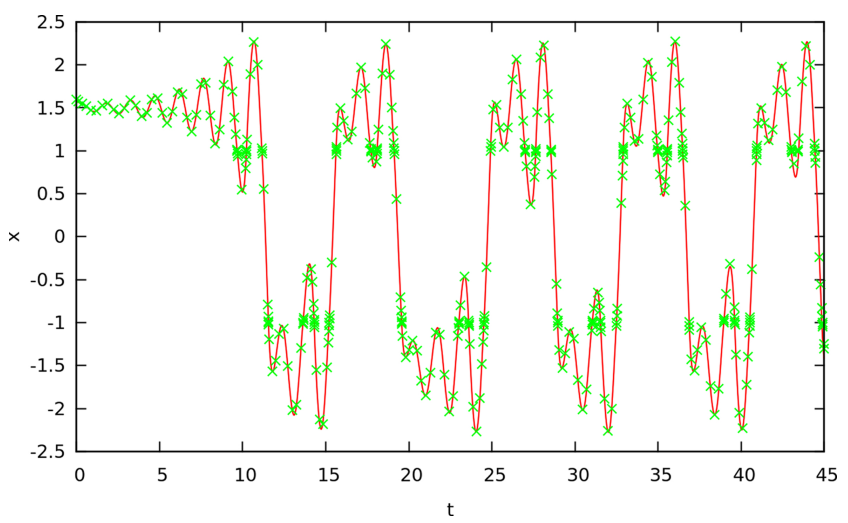

Figura 17: Curvas de $x$ em função de $t$ obtidos com o aplicativo Circuit of Chua and Chaos (linhas) comparadas às curvas obtidas com o programa MATDS (pontos na forma de $x$ ), os parâmetros mostrados na Eq. (47). Os resultados são coincidentes.

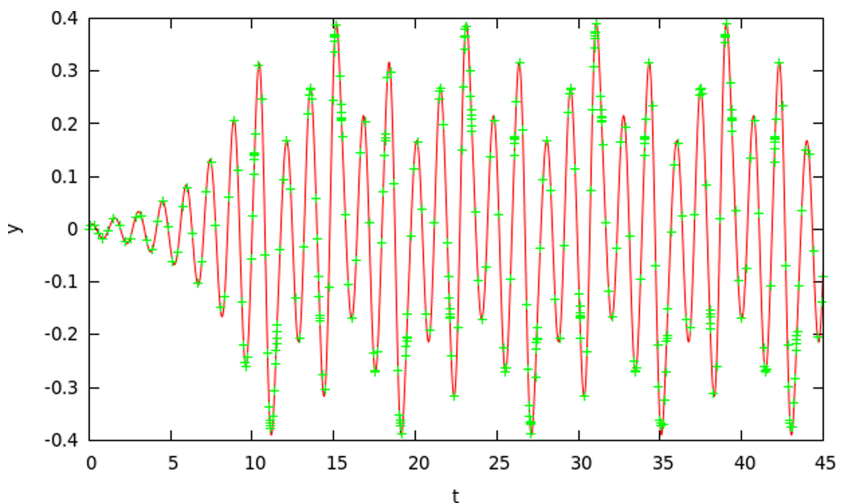

Figura 18: Curvas de $y$ em função de $t$ obtidos com o aplicativo Circuit of Chua and Chaos (linhas) comparadas às curvas obtidas com o programa MATDS (pontos na forma de $x$ ), os parâmetros mostrados na Eq. (47). Os resultados são coincidentes. 


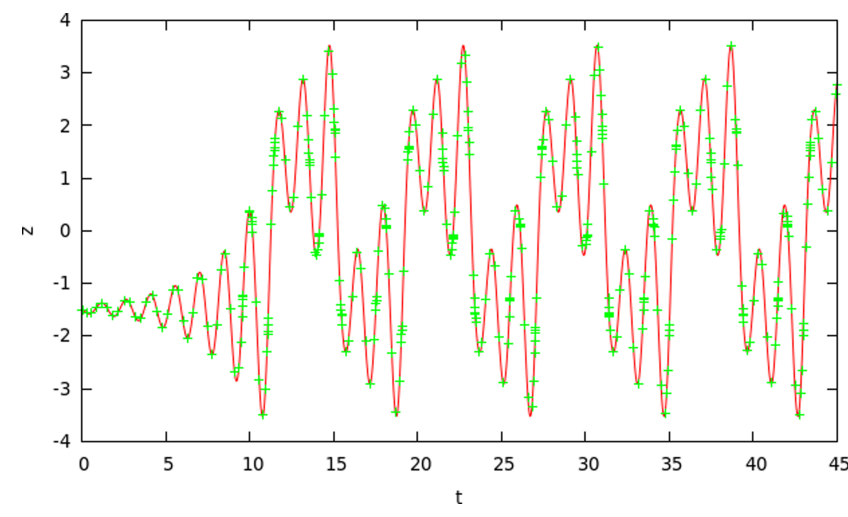

Figura 19: Curvas de $z$ em função de $t$ obtidos com $o$ aplicativo Circuit of Chua and Chaos (linhas) comparadas às curvas obtidas com o programa MATDS (pontos na forma de $x$ ), os parâmetros mostrados na Eq. (47). Os resultados são coincidentes.

nologias, tais como: o número de usuários ativos do Android ultrapassou a quantidade de um bilhão de usuários em 2014: ${ }^{2}$ o custo dos dispositivos é menor; os dispositivos possuem tela sensível ao toque; os dispositivos apresentam maior comodidade de manuseio (ocupam pouco espaço, não apresentam superaquecimento, apresentam um menor consumo de energia elétrica).

O aplicativo Circuit of Chua and Chaos foi desenvolvido para o estudo de um sistema que apresenta um certo grau de complexidade, uma vez que as soluções podem ser caóticas. O aplicativo mostrouse eficiente na obtenção de expoentes de Lyapunov; projeções das trajetórias nos planos $x y, x z$ e $y z$; e gráficos de $x(t), y(t)$ e $z(t)$. Os resultados do aplicativo foram exportados para aquivos txt e comparados com os resultados obtidos com o programa MATDS, utilizado por muitos dos pesquisadores que investigam o circuito de Chua-Matsumoto, inclusive o próprio Leon Chua, que dá nome ao circuito, como pode ser visto na Ref. 9]. A partir da comparação, verificou-se que os resultados obtidos com o aplicativo coadunam com os obtidos com o MATDS.

Pretende-se desenvolver outros programas de simulação para plataforma Android, de forma a cobrir o conteúdo da disciplina de sistemas dinâmicos que normalmente é ministrado em um curso regular de graduação.

\footnotetext{
$\overline{2 \text { ver http://www.techspot.com/news/ }}$
}

\section{Referências}

[1] Luiz Henrique Alves Monteiro, Sistemas Dinâmicos (Ed. Livraria da Física, São Paulo, 2011), $3^{\text {a }}$ ed.

[2] Stephen Lynch, Dynamical Systems with Applications using MATLAB囚 (Birkhäuser, Basileia, 2014).

[3] Ahmed G. Radwan and Mohammed E. Fouda, On the Mathematical Modeling of Memristor, Memcapacitor, and Meminductor (Springer International Publishing, Suiça 2015).

[4] T. Matsumoto, IEEE Trans. on Circ. \& Syst. 31, 1055 (1984).

[5] T. Matsumoto, L.O. Chua and M. Komuro, IEEE Trans. on Circ. \& Syst. 32, 797 (1985).

[6] T. Matsumoto, L.O. Chua and M. Komuro, Journal Circuit Theory and Appl. 14, 117 (1986).

[7] M.P. Kennedy, Frequenz 46, 66 (1992).

[8] J.M. Cruz and L.O. Chua, IEEE Trans. on Circ. \& Syst. 46, 985 (1992).

[9] M. Itoh and L.O. Chua, Int. J. Bif. and Chaos 18, 3183 (2008).

[10] B. Muthuswamy, Int. J. Bif. and Chaos 453, 1335 (2010).

[11] B. Muthuswamy and L.O. Chua, Int. J. Bif. and Chaos 20, 1567 (2009).

[12] L.O. Chua, IEEE Trans. on Circ. Theory 5, 507 (1971).

[13] D.B. Strukov, G.S. Snider, D.R. Stewart and S.R. Williams, Nature 453, 80 (2008).

[14] A. Buscarino, L. Fortuna, M. Frasca and L.V. Gambuzza, Chaos: Interdiscip. J. Nonlinear Sci. 22, 023136 (2012).

[15] L.O. Chua, M. Komuro and T. Matsumoto, IEEE Trans. on Circ. and Systems 33, 1072 (1986).

[16] L.P. Shil'nikov, Dokl. Akad. Nauk SSSR 160, 558 (1965).

[17] Richard L. Burden e J. Douglas Faires, Análise Numérica (CENGAGE Learning, São Paulo, 2008) $8^{\mathrm{a}}$ ed.

[18] A. Wolf, J.B. Swift, H.L. Swinney and J.A. Vastano, Physica D 16, 285 (1985). 